\title{
The Technological Adolescent Age Transition: A Boundary to Estimate the Last Factor of the Drake Equation
}

\author{
Guillermo A. Lemarchand \\ Instituto Argentino de Radioastronomía (CONICET) and Centro de \\ Estudios Avanzados(UBA).E-mail:lemar@cea.uba.ar
}

\begin{abstract}
Based on the study of several long-term societal indicators we estimate the phase transition between the "Technological Adolescent Age" (TAA) and the "Technological Mature Age" (TMA). Assuming the "Principle of Mediocrity" and using the Drake Equation we estimate a lower threshold for the number of technological civilizations in the galaxy.
\end{abstract}

\section{Introduction}

Sagan (1980) defined the TAA as the stage in which an intelligent species has the capability to become extinct due to technological misuse (e.g., global war), environmental degradation of the home planet (e.g., global warming, overpopulation, etc.), or simply by the bad distribution of physical, educational and economical resources (difference between the degree of development among developed and developing societies). Sagan (1980) considered that the present human civilization is passing through the TAA.

We present a semi-empirical approach to estimate the period of time that will be taken by humankind to pass through TAA into a TMA in which we would learn how to live in harmony with the members of our species and the environment and learn how to generate power efficiently. The last stage is an imperative requirement needed to extend the lifetime of our civilization. This transition may be a typical evolutionary stage for all the hypothetical galactic civilizations (Principle of Mediocrity).

In 1961 Frank Drake (Pearman 1963) proposed an equation to estimate the number of technological civilizations in our galaxy. Several simulations assigning different values to each factor of the Drake Equation, showed that the number of technological galactic civilizations $(N)$ has a strong dependence with the last factor of the equation, $L$, or the lifetime of a civilization in years (Kreifeldt 1973; Oliver 1975). Most of the authors would agree that the possible number of technological civilizations in the galaxy would be close to $N \simeq(0.1 \times L)$.

\section{The Determination of the TAA Transition}

Several very long-term biological and ecological studies have shown that different species on Earth, emerge, develop and become extinct with similar evolutionary patterns (Charnov 1993; Gurney \& Nisbet 1998). Human species may be not an 
exception. Our hypothesis is that we are living in a very special moment of our species history, the transition from the TAA to the MTA (Lemarchand 2000). So, if we keep the same inter-individual dynamical relations among the members of the human society that were present during - at least - the last 12000 years (since the emergence of agriculture and cities), we have a high probability of becoming extinct in the next 150-200 years. On the other hand, if we learn how to shift societal behavior and change the inter-individual dynamical relations, we may probably extend our societal life expectancy.

In order to estimate the period of time of this TAA transition we have used three different societal indicators: (1) the human demographic transition, (2) the distribution of deadly quarrels during the last five centuries and (3) the diffusion of democracies within nations (considered as a disembodied technology of government) between 1800-2000. The available temporal series comes from a few hundred years (e.g., world democracies) to several thousand years (e.g., the evolution of human population). We have found that the temporal series analyzed for the first two societal indicators follow a Self-Organized Criticality (SOC) system with a scale free behavior, a characteristic presented in most of the complex systems (Bak 1996; Jensen 1998). On the other hand, the third indicator shows a logistic growth pattern, similar to those found in the diffusion of new technologies in a close market (Fisher \& Pry 1971).

The data corresponding to the three indicators shows similar phase-transition patterns starting after the World War II (WWII) and ending by the year 2100 . Within this particular period of our human species history, we have developed sophisticated war-technologies, so efficient, that we may become extinct at a rate of 500 million people per hour. We may destroy the natural balance of our biosphere by the industrial pollution, the greenhouse effect and other environmental degradation activities generated by the human behavior. We may cause a world population explosion that may rapidly exhaust the natural resources or we may increase the gap between developed and underdeveloped societies in such a way to generate the collapse of different regions of our home planet. All these examples may be described in the long term by several mathematical approaches that show the emergence of patterns very similar to those that appear in the ecological dynamics of other species.

\section{Empirical Results and Data Analysis}

\subsection{The Demographic Transition}

We have used Kapitza's (1996) mathematical model of the world population growth that shows a blow-up and self-similar regime. We found that this model and the available historical data of the global human population exhibits a SOC behavior. Another intrinsic property of the model is the so-called Demographic Transition or the well established change in the pattern of growth of all populations, when they reach a certain stage and rate of development. This transition has been experienced by all developed countries; it began there at the end of $18^{\text {th }}$ Century with the Industrial Revolution. At the present, we are at the height of the transition on a global scale. Following the mathematical model, the absolute rate of global population growth is expected to peak in the year 2007 , but the relative growth rate reached its maximum value of 1.7 per year in 
Average Annual Increase of World Population

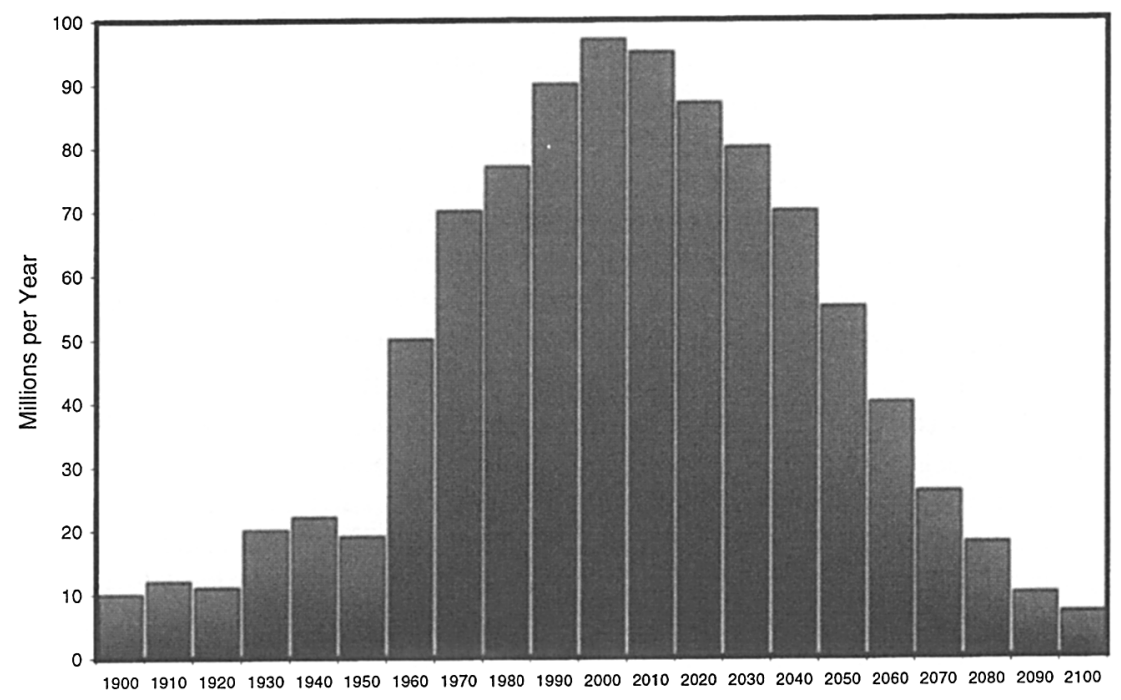

Figure 1. Average annual increase of world population (in millions per year). Based on data provided by T. Merrick, World Population in Transition, Population Bulletin

1989. The equations show that -for a planetary behavior- the human population started with its demographic transition by 1960 and would end by 2050 . After that a new reproductive regime is expected to appear, different to the previous one that dominated the dynamics of the last 12000 years (since the emergence of agriculture and the cities).

\subsection{The Distribution of Deadly Quarrels During $1500-2000$}

Richardson (1945) discovered that the distributions of wars over time follow a power-law (using data from 1800 to 1930). This is a characteristic of all SOC systems. In one way, this shows that the dynamics on inter-human violence is governed by the same type of processes present in the human population growth dynamics and in a great variety of other complex systems examples (Bak 1996).

Although there is a pattern in the dynamics of inter-human violence, following a SOC system, we also have to take into account the evolution of the technologies of war against time. We have worked with a coefficient of lethality in order to normalize the evolution of weapon technologies from the sword in 400 B.C. to the atomic bombs at the end of the twentieth Century. We have also analyzed the distribution of wars between 1495-2000, extending the original Richardson work to 500 years of data.

We introduced a definition of intensity of a war, $I$, as the ratio of battle deaths to the population at the time of the war (Levy 1983). We have represented the distribution of the number of battles $N_{c}$ against the intensity $I$. However, when considering distributions that may exhibit SOC it is preferable to use noncumulative data. An equivalent approach is to take the mathematical derivative 


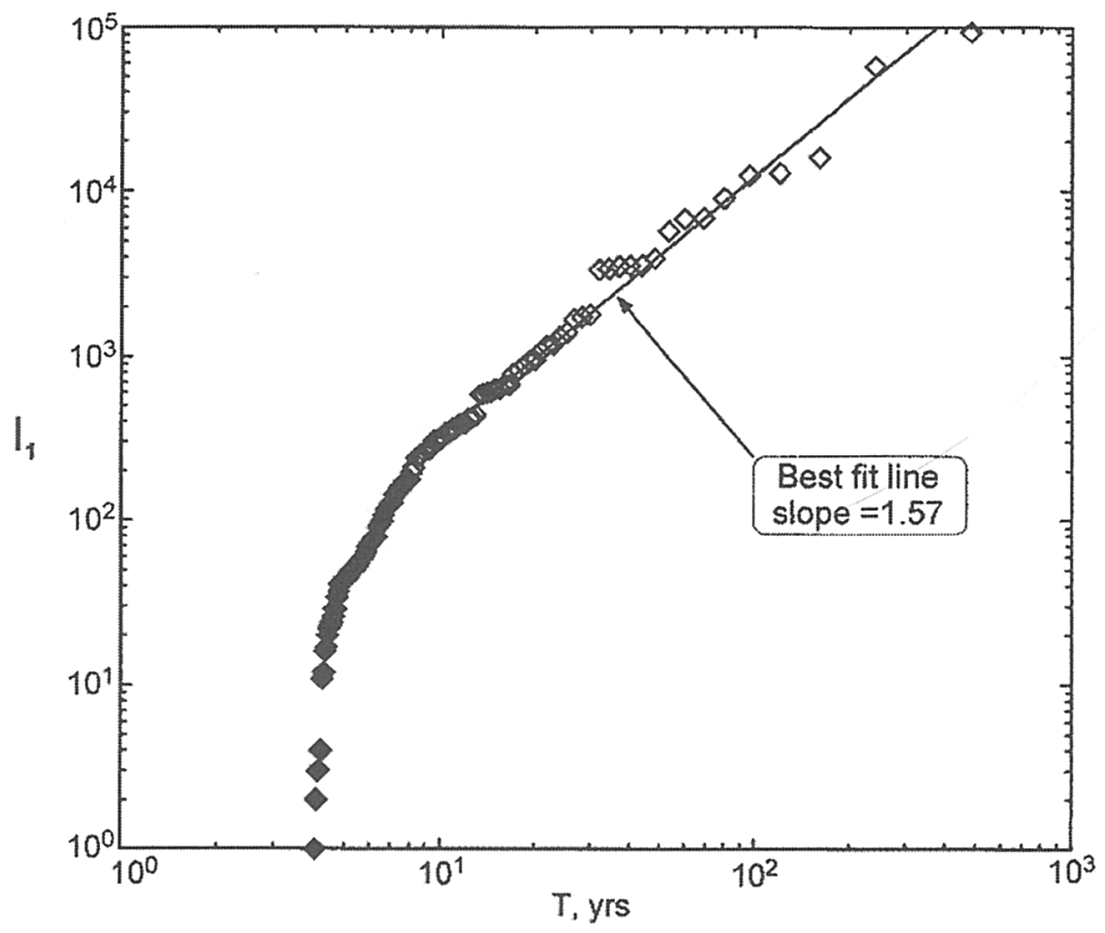

Figure 2. Dependence of the war intensity $I$ on the return period $T$. The larger wars correlate well with the fractal relation $I=C_{1} T^{D}$ taking $D=1.57$. $I$ is the ratio of battle deaths to the population in millions at the time of the war and $C_{1}$ is a constant (Roberts \& Turcolotte 1998). 


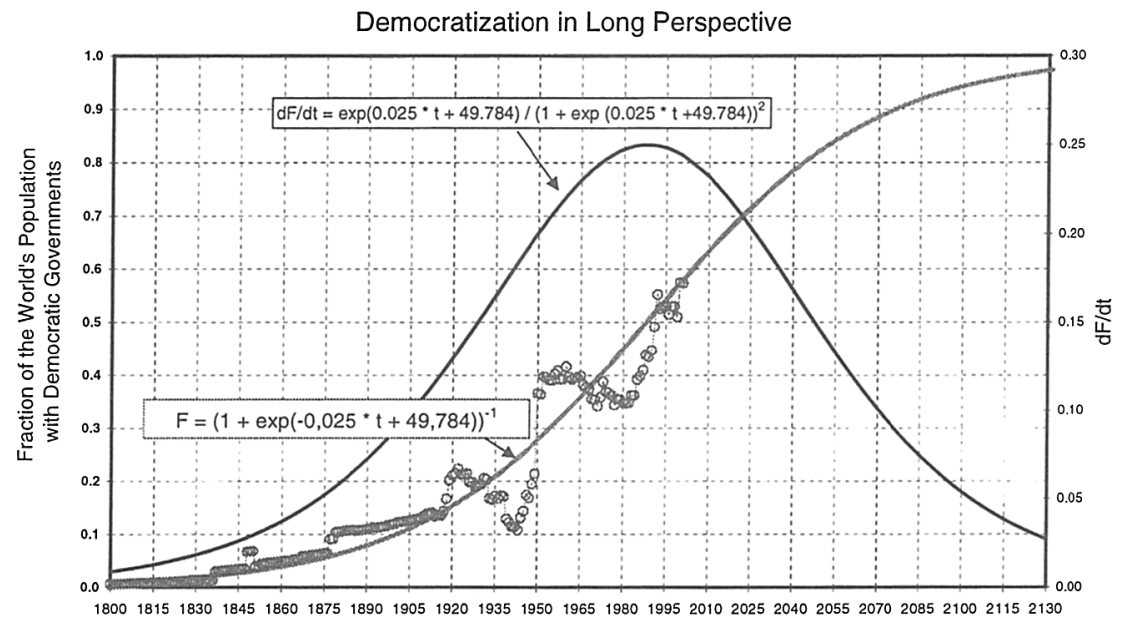

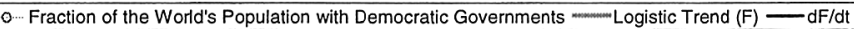

Figure 3. Distribution of democracies represented against time (1800-2000). Here, we calculated the fraction of the world population under democratic governments with a score over 6 points, according to POLITY IV data-base. If we consider the democracy as a disembodied technology of government we see that diffuses against time as any other technology in a close market, following a logistic type growth. $\mathrm{F}$ is the fraction of the world population that is under a democratic government at time $t$ and may be represented by a logistic equation that describes the evolution and diffusion of democracies against time. The bell-shape curve is the density function $(\mathrm{dF} / \mathrm{dt})$. The takeover time $(\mathrm{dt})$ is defined as the time required for the technology to increase from $\mathrm{F}=0.10$ to $\mathrm{F}=0.90$. In our case $\mathrm{dt}=176$ years.

of the cumulative distribution with respect to intensity $d N_{c} / d I$. Here the fractal dimension resulted in 1.28. An alternative approach to the analysis of this data is in terms of return periods $T$, the time we should wait to have an event of intensity $I$. When we consider these distributions of deadly quarrels using normalized values of technologies, we found a correlation between the coefficient of lethality and the slope of the SOC distribution. A rough extrapolation of this analysis shows that - using 500 years of deadly quarrels data - we would need to wait between 30 to 500 years in order to generate a violent event at which the whole human population will disappear. As a check, we have looked at a couple of auxiliary indicators of the war processes: (a) the distribution of the destructive power available in nuclear arsenals per capita or the number of tons of TNT per person in the world during 1954-2000 and (b) the evolution of world military expenditures during 1950-2000. Both indicators show an extraordinary coincidence in their distributions with the demographic transition period with a highest peak around 1980-1990. 


\subsection{The Diffusion of Democracies within Nations 1800-2000}

We have analyzed the evolution of this societal indicator in order to understand the time constants at which societies organized themselves in order to produce changes in a macro-behavior level. Democracy is a mechanism of collective choice and a form of social organization that can be considered a superior substitute for other such mechanisms or forms of organization. In this way, democracy may be considered as a disembodied technology of government and social organization. As such, as well as any other embodied or disembodied technology, democracy may be expected to grow, or diffuse, over time, amongst the world's population, and the hypothesis posed in the present study is that the growth follow a regular pattern, according with the Fischer \& Pry (1971) substitution model of technological change.

To test this hypothesis we have used, for the period 1800-2001, the list of national democracies (www.bsos.umd.edu/cidcm/polity) provided in the POLITY IV dataset. The Polity IV survey covers all independent members of the international system, those that have attained independence by 1975 and whose population exceeded one million by the mid-1990s. It gives, for each such polity, an annual score of institutional democracy, on a scale ranging from zero to ten. Using this data set, we calculate the fraction of the world population at each year of those polities that have reached at least 6 points in the POLITY IV scores. The results show that the dynamics of the diffusion of democracies among the world population follows a logistic growth curve. The calculation of the mathematical derivative of the logistic curve also shows a bell-shape curve centered at the year 1988. This is similar to the distribution found for the demographic transition data and the deadly quarrels analysis.

\section{Conclusions}

With the invention of the technologies of mass destruction after WWII we have - for the first time in the human history - the technological capability to annihilate our species and most of the life forms on Planet Earth. The long-term evolution of the three different societal indicators analyzed, showed a transition that started after the WWII and that may end after the middle of XXI Century. If we want to avoid self-extinction we must change the rules of the inter-human interaction within the limits of this particular period of time, defined as TAA.

After the WWII our terrestrial civilization reached the technological capability for interstellar communication via electromagnetic waves (radio and laser signals). In a broad sense, the bottleneck for the evolution of any technological civilization in the galaxy would be the TAA.

If we assume the so-called Principle of Mediocrity (Hoerner 1961) that proposes that our planetary system and our civilization are about average and that life and intelligence will develop by the same rules of natural selection wherever the proper surroundings and the needed time are given, we may also assume that the average lower boundary for a technological civilization lifetime with interstellar communication capabilities would be close to $\mathrm{L} \simeq 150$ to $200 \mathrm{yrs}$. If this is so, we may use the Drake Equation to determine the lower limit of the number of technological civilizations in our galaxy as $\mathrm{N} \simeq(0.1 \times \mathrm{L})=15$ to 20 . 
Acknowledgments. This research is supported by a Foundation for the Future research grant. My participation in this meeting was possible thanks to the generosity of Allen Tough and an IAU travel grant.

\section{References}

Bak, P. 1996, How Nature Works, (New York: Springer-Verlag)

Charnov, E. L. 1993, Life History Invariants, (Oxford: Oxford University Press)

Fisher, J. C., \& Pry, R. H. 1971, Tech. Forecast. \& Social Change, 3, 75

Gurney, W. S. C., \& Nisbet, R. M. 1998, Ecological Dynamics, (Oxford: Oxford University Press)

Hoerner, S. von 1961, Science, 134, 1839

Jensen, H. J. 1998, Self-Organized Criticality, (Cambridge: Camb. Univ. Press)

Kapitza, S. P. 1996, Physics-Uspekhi, 29, 57

Kreifeldt, J. G. 1973, Icarus, 14, 419

Lemarchand, G. A. 2000, in When SETI Succeeds: The Impact of HighInformation Contact, ed. A. Tough, (Bellevue: The Foundation for the Future), 153

Levy, J. S. 1983, War in the Modern Great Powers, (Lexington: Univ. of Kent.) Oliver, B. 1975, Icarus, 25, 360

Pearman, J. P. T. 1963, in Interstellar Communication: The Search for Extraterrestrial Life, ed. A. G. W. Cameron, (New York: W. A. Benjamin)

Richardson, L. F. 1945, Nature, 155, 610

Roberts, D. C., \& Turcolotte, D. L. 1998, Fractals, 6, 353

Sagan, C. 1980, Cosmos, (New York: Random House) 